Case Report

\title{
Is It Necessary to Specifically Define the Cause of Surgically Treated Biliary Tract Infections? A Rare Case of Raoultella planticola Cholecystitis and Literature Review
}

\author{
Suat Can Ulukent, ${ }^{1}$ İnanc Samil Sarici, ${ }^{1}$ Nuri Alper Sahbaz, ${ }^{1}$ \\ Yigit Mehmet Ozgun, ${ }^{1}$ Ozlem Akca, ${ }^{1}$ and Kamuran Sanlı ${ }^{2}$ \\ ${ }^{1}$ Kanuni Sultan Suleyman Training and Research Hospital, Department of General Surgery, Istanbul, Turkey \\ ${ }^{2}$ Kanuni Sultan Suleyman Training and Research Hospital, Department of Clinical Microbiology, Istanbul, Turkey \\ Correspondence should be addressed to İnanc Samil Sarici; issarici2015@gmail.com
}

Received 18 October 2016; Revised 9 February 2017; Accepted 16 February 2017; Published 7 May 2017

Academic Editor: Alexandre Rodrigues Marra

Copyright (C) 2017 Suat Can Ulukent et al. This is an open access article distributed under the Creative Commons Attribution License, which permits unrestricted use, distribution, and reproduction in any medium, provided the original work is properly cited.

Raoultella planticola is an aquatic and soil organism that does not notoriously cause invasive infections in humans. Infections in the literature are limited only in case reports. We present a very rare case of $R$. planticola cholecystitis. A 71-year-old female patient with abdominal pain was diagnosed with acute cholecystitis. Patient received intravenous antibiotic treatment, but the treatment failed and the patient underwent an open cholecystectomy. The final pathological result was gangrenous cholecystitis complicated with R. planticola. Eventually, the patient recovered with appropriate antimicrobial therapy. Patients with acute cholecystitis are usually treated without any microbiological sampling and antibiotic treatment is started empirically. To date, there have only been 5 reported biliary system related $R$. planticola infections in humans. We believe that Raoultella species might be a more frequent agent than usually thought, especially in resistant cholecystitis cases. Resistant strains should be considered as a possible causative organism when the patient's condition worsened despite proper antimicrobial therapy. It should be considered safe to send microbiological samples for culture and specifically define the causative microorganisms even in the setting of a cholecystectomized patient.

\section{Introduction}

Raoultella planticola is an aquatic and soil organism that does not notoriously cause invasive infections in humans. Raoultella planticola infections are limited only in case reports. There have only been 5 reported cases of biliary tract related infections (cholecystitis, cholangitis, and pancreatitis) described so far in the English literature. Hereby, we report a case of cholecystitis complicated by $R$. planticola.

\section{Case}

A 71-year-old female patient is presented to the emergency department with the chief complaint of abdominal pain. She described a 1-week history of worsening right upper quadrant pain with nausea and vomiting. Her physical examination body temperature $37.5^{\circ} \mathrm{C}$, pulse $90 / \mathrm{min}$, blood pressure $120 / 80 \mathrm{mmHg}$, and respiratory rate $15 / \mathrm{min}$. A slight distension and a tenderness in the right hypochondrium area were detected in the abdominal examination; abdominal defense and rebound phenomenon were absent. Her medical history revealed geophagia during childhood and a previous long-term dental treatment. She did not have diabetes or any prior surgeries.

White Blood Cell (WBC) count was $22.9 \times 10^{9} / \mathrm{L}$ [normal: $3.7 \times 10^{9} / \mathrm{L}-10 \times 10^{9} / \mathrm{L}$ ], and C-reactive protein $(\mathrm{CRP})$ was $150(\mathrm{NV}<5)$. Ultrasound (USG) scan revealed a distended gall bladder with a thickened wall and multiple stones. The patient was diagnosed as acute cholecystitis and admitted to the surgical ward.

She received 3 days of intravenous (IV) cefazolin $2 \times$ 1 gr/day. At the end of the 3rd day, her symptoms improved, 
TABLE 1: Biliary tract related infections caused by $R$. planticola, reported in the literature.

\begin{tabular}{lcccccc}
\hline Author & Patient & Clinical manifestation & Comorbidity & $\begin{array}{c}\text { Trauma/invasive } \\
\text { procedure }\end{array}$ & $\begin{array}{c}\text { Immune } \\
\text { deficiency }\end{array}$ & Treatment \\
\hline Alves et al. & $45 /$ male & $\begin{array}{c}\text { Pancreatitis and } \\
\text { retroperitoneal abscess }\end{array}$ & Alcoholism, pneumonia & No & Yes & Imipenem, amikacin \\
\hline Yokota et al. & $65 /$ male & Cholangitis & $\begin{array}{c}\text { Adenocarcinoma of the } \\
\text { neck }\end{array}$ & ERCP* & Yes & Piperacillin, tazobactam \\
\hline Teo et al. & $62 /$ female & Acute cholecystitis & Celiac disease & No & No & Amoxicillin-clavulanate \\
\hline Salmaggi et al. & $70 /$ male & Acute cholecystitis & $\begin{array}{c}\text { Pancreatic } \\
\text { adenocarcinoma }\end{array}$ & $\begin{array}{c}\text { ERCP, biliary } \\
\text { stenting }\end{array}$ & Yes & $\begin{array}{c}\text { Ciprofloxacin, } \\
\text { metronidazole }\end{array}$ \\
\hline Ershadi et al. & $49 /$ male & Acute cholecystitis & $\begin{array}{c}\text { Alcoholism, alcoholic } \\
\text { cirrhosis, diabetes } \\
\text { mellitus }\end{array}$ & $\begin{array}{c}\text { Percutaneous } \\
\text { cholecystostomy }\end{array}$ & Yes & Tigecycline \\
\hline
\end{tabular}

*ERCP: endoscopic retrograde cholangiopancreatography.

but WBC and CRP levels remained high. On the 7th day of her treatment, her symptoms resolved and WBC levels normalized, but paradoxically CRP levels still remained high and control ultrasound revealed an increase in the thickness of the gall bladder wall and pericholecystic fluid collection. According to these findings, surgery was planned. During exploration, the gall bladder was found to be buried in the omentum with multiple foci of closed perforation. Cholecystectomy was performed and no complications occurred during the postoperative period. Cultures of microbiological sample were taken during the operation. The sample was cultivated on EMB and sheep blood agar with standard loop as the inoculant. Gram-negative bacilli grew on both culture media to a density of $>100,000 \mathrm{CFU} / \mathrm{mL}$. The isolate was analyzed by matrix-assisted laser desorption/ionization time-offlight mass spectrometry using the VITEK MS (bioMérieux, Marcy l'Étoile, France) system and identified as R. planticola. Antimicrobial susceptibility testing was performed using the VITEK-2 compact system which was sensitive to cefazolin, ampicillin/sulbactam, ceftriaxone, ciprofloxacin, gentamicin, ceftazidime, tobramycin, and levofloxacin and resistant to ampicillin, cefuroxime, imipenem, trimetho$\mathrm{prim} /$ sulfamethoxazole, amoxicillin/clavulanic acid, cefuroxime axetil, and nitrofurantoin. According to the culture results, the ampicillin/sulbactam regimen was continued for 10 days and the patient was discharged with normal CRP levels and no complications. The final pathological result was gangrenous cholecystitis. In the 3rd postoperative month of follow-up, all the blood stats, USG, and magnetic resonance cholangiopancreatography (MRCP) images were normal.

\section{Discussion}

Raoultella planticola is a nonmotile, aerobic, Gram-negative bacillus. It was first described by Bagley et al. [1] as Klebsiella planticola in 1981 within environmental specimens and by Ferragut et al. [2] in 1983 as Klebsiella trevisanii with deoxyribonucleic acid (DNA) hybridization technique in soil and water specimens. Because of the DNA sequence similarities, in 1986, both microorganisms were merged within one species, Klebsiella planticola, by Gavini et al. [3]. R. planticola carries a $\beta$-lactamase that makes this agent naturally resistant to several antimicrobial agents.

To date, there have only been 5 reported biliary system related infections in humans (Table 1). Alves et al. [4] in 2007 reported a case of acute pancreatitis and retroperitoneal abscess in a 45-year-old male. Yokota et al. [5] described a 65year-old man developing septic shock and cholangitis caused by $R$. planticola in 2011. In 2012, Teo et al. [6] reported acute cholecystitis in a 62-year-old woman. In 2014, Salmaggi et al. [7] described a 70-year-old man with chronic obstructive pulmonary disease and pancreatic adenocarcinoma complicated with $R$. planticola-associated cholangitis and, in the same year, Ershadi et al. [8] reported a 49-year-old man presenting with acute cholecystitis with past medical history of alcohol abuse, alcoholic cirrhosis, and diabetes mellitus. Most of the infected cases in literature either have significant comorbidities or have a history of trauma or an invasive procedure, but there is no trauma history or comorbidity seen in our case.

Antibiotic treatment is started empirically with agents covering enterococci and Enterobacteriaceae, including Escherichia coli. Generally, acute cholecystitis cases are expected to be clinically regressed after parenteral antibiotic treatment. If the clinical regression does not occur within 2 days, it is considered resistant cholecystitis. We believe that Raoultella species might be a more frequent agent then usually thought, especially in resistant cholecystitis cases. $R$. planticola has the ability to become antibiotic-resistant trough plasmid genes. Most of the cases reported in the literature are severe cases of infection or sepsis. Therefore, resistant strains should be considered as a possible causative organism when the patient's condition worsens despite proper antimicrobial therapy.

\section{Conclusion}

Potential risk factors for $R$. planticola include invasive medical procedures, immunocompromised patients, significant comorbidities, and trauma with soil contamination.

We believe that resistant strains may also cause serious infections in the absence of these predisposing factors. 
Despite the fact that our patient did not have any risk factors and had severe case of cholecystitis, it is still prudent to consider $R$. planticola as a possible causative agent among patients with these risk factors. Given its resemblance to Klebsiella species, this bacterium has the potential to become multidrug resistant and cause increased numbers of mortality and morbidity. In order to prevent this, it should be viewed as an invasive organism, requiring proper treatment, rather than a noninfectious member of the flora. Hence, it should be considered safe practice to send microbiological samples for culture and specifically define the causative microorganisms even in the setting of a cholecystectomized patient.

\section{Conflicts of Interest}

The authors declare that there are no conflicts of interest regarding the publication of this paper.

\section{References}

[1] S. T. Bagley, R. J. Seidler, and D. J. Brenner, "Klebsiella planticola sp. nov.: A new species of enterobacteriaceae found primarily in nonclinical environments," Current Microbiology, vol. 6, no. 2, pp. 105-109, 1981.

[2] C. Ferragut, D. Izard, and F. Gavini, "Klebsiella trevisanii: A new species from water and soil," International Journal of Systematic Bacteriology, vol. 33, no. 2, pp. 133-142, 1983.

[3] F. Gavini, D. Izard, and P. A. D. Grimont, "Priority of Klebsiella planticola Bagley, Seidler, and Brenner 1982 over Klebsiella trevisanii Ferragut, Izard, Gavini, Kersters, De Ley, and Leclerc 1983," International Journal of Systematic Bacteriology, vol. 36, no. 3, pp. 486-488, 1986.

[4] M. S. Alves, L. W. Riley, and B. M. Moreira, "A case of severe pancreatitis complicated by Raoultella planticola infection," Journal of Medical Microbiology, vol. 56, no. 5, pp. 696-698, 2007.

[5] K. Yokota, H. Gomi, Y. Miura, K. Sugano, and Y. Morisawa, "Cholangitis with septic shock caused by raoultella planticola," Journal of Medical Microbiology, vol. 61, no. 3, pp. 446-449, 2012.

[6] I. Teo, J. Wild, S. Ray, and D. Chadwick, "A Rare Case of Cholecystitis Caused by Raoultella planticola," Case Reports in Medicine, Article ID 601641, 2012.

[7] C. Salmaggi, F. Ancona, J. Olivetti, G. Pagliula, and G. A. Ramirez, "Raoultella planticola-associated cholangitis and sepsis: A case report and literature review," QJM, vol. 107, no. 11, pp. 911-913, 2014.

[8] A. Ershadi, E. Weiss, E. Verduzco, D. Chia, and M. Sadigh, "Emerging pathogen: a case and review of Raoultella planticola," Infection, vol. 42, no. 6, pp. 1043-1046, 2014. 


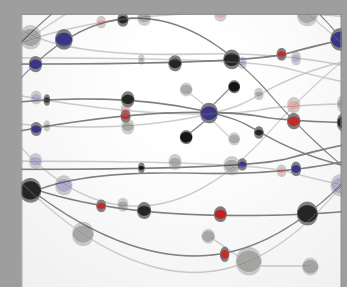

The Scientific World Journal
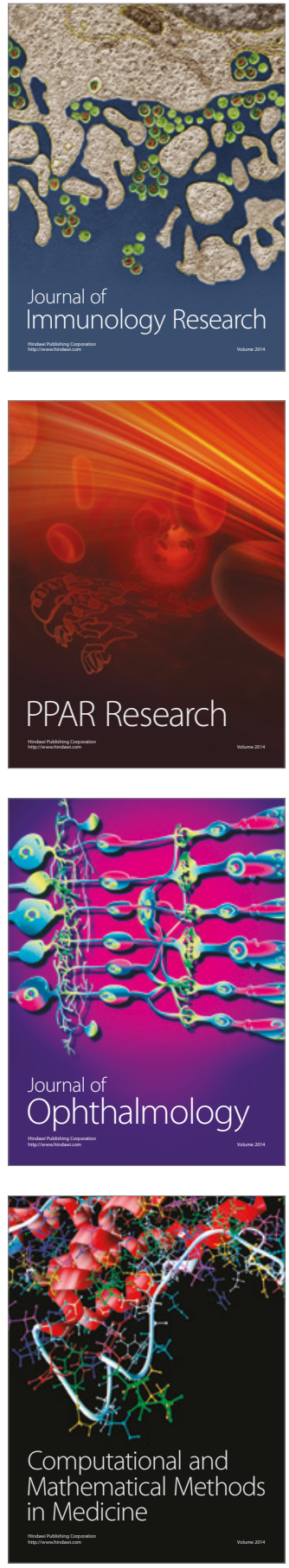

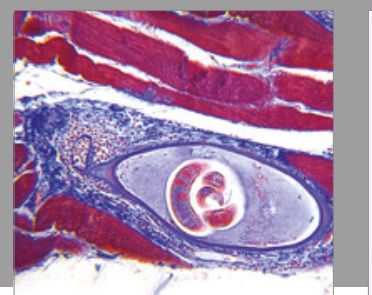

Gastroenterology Research and Practice
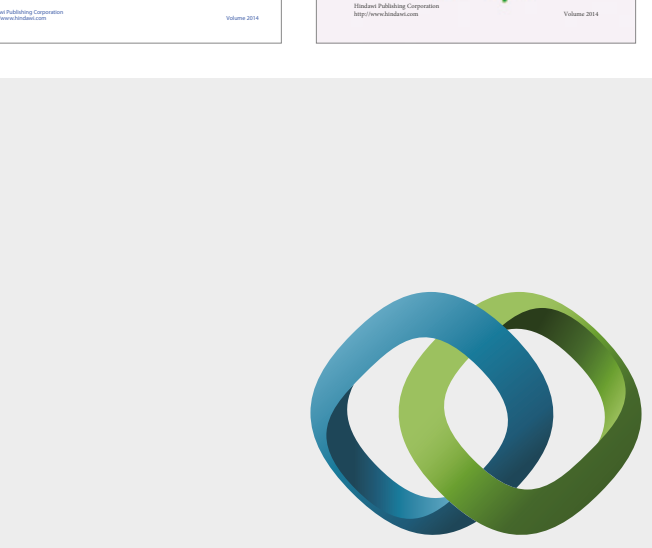

\section{Hindawi}

Submit your manuscripts at

https://www.hindawi.com
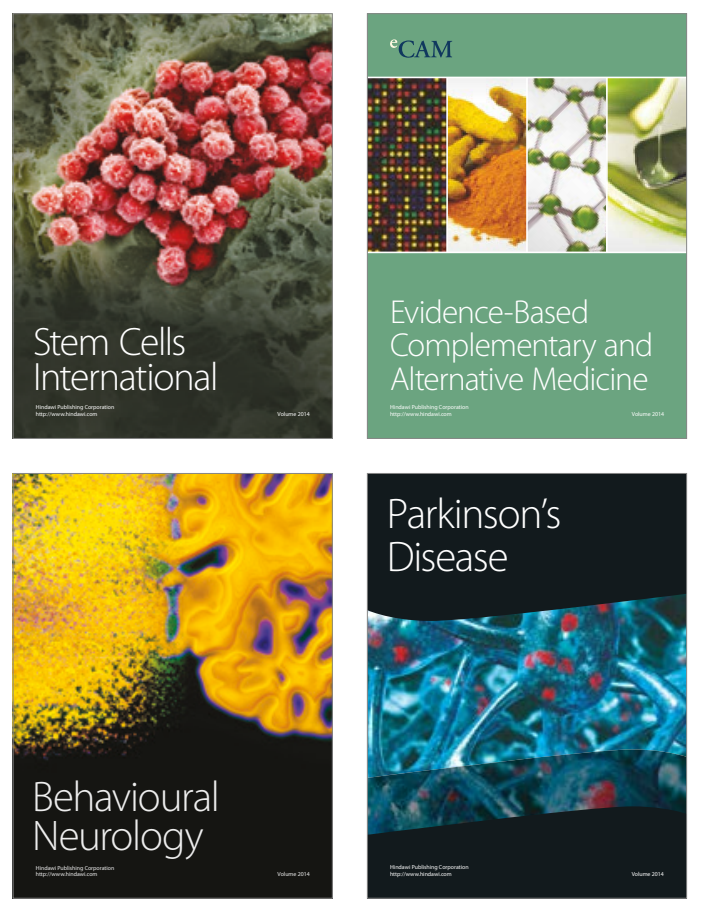
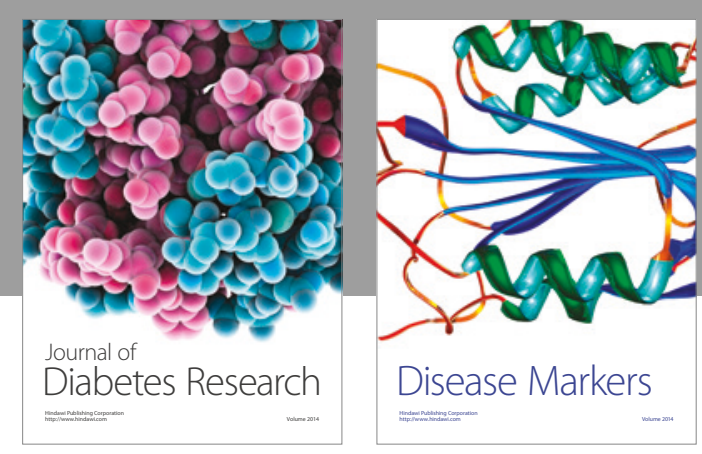

Disease Markers
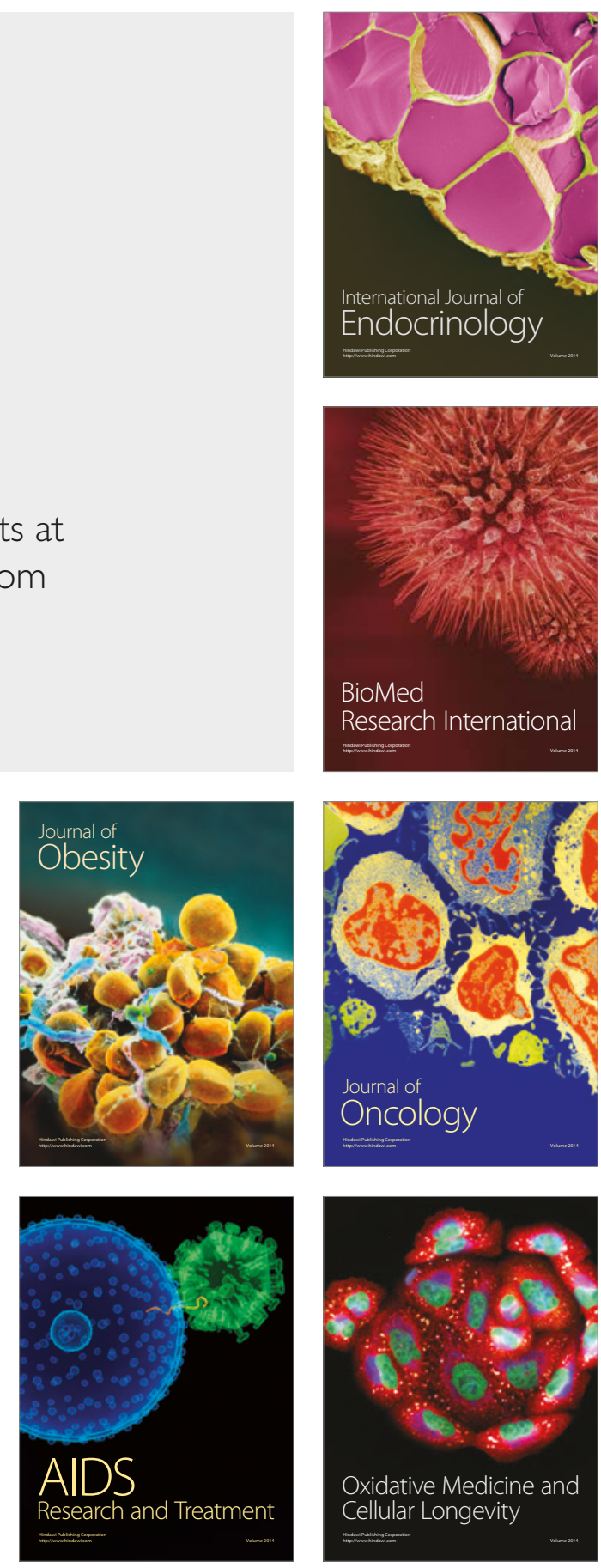